\title{
A New Method for Reliability Evaluation of Wireless Sensor Networks Based on Fuzzy Neural Network
}

\author{
https://doi.org/10.3991/ijoe.v14i10.9315 \\ Jianjun $\mathrm{Xu}$ \\ Nanjing Normal University Taizhou College, JiangsuTaizhou, China \\ x_j_j1981@126.com
}

\begin{abstract}
In this paper, a reliability evaluation model based on fuzzy neural network is proposed to evaluate the reliability of wireless sensor networks without a unified standard. Firstly, the reliability is analyzed from the point of view of topology structure, protocol stack structure and reliability mechanism of wireless sensor network, and the performance indexes that affect the reliability are extracted. Secondly, some performance indexes are screened out, and the standard value matrix of reliability evaluation for index fuzzy quantization is established. The sample data is generated by interpolation, and the reliability evaluation model based on fuzzy neural network is established. The neural network model takes the selected index values as input, and outputs are the reliability of the wireless sensor network. The simulation results show that the evaluation model is basically consistent with the actual situation, and it can evaluate the wireless sensor network from the system level.
\end{abstract}

Keywords-Wireless sensor networks; fuzzy neural network; reliability evaluation; performance indexes

\section{Introduction}

Wireless sensor network (WSN) has the advantages of convenient installation, automatic networking, wireless communication and low cost, so it has been widely used in logistics, industrial monitoring, smart grid [1], smart home [2] and other fields. In order to obtain better quality of network intelligence services, the reliability of WSN has received attention. For a WSN to be constructed, the performance indexes such as network delay, network lifetime, fault tolerance and redundancy of the network, and environmental interference need to be considered in advance. Each performance index doesn't exist in isolation, but affects each other and restricts each other. By establishing the relationship between each performance index and the reliability of the sensor network, it can be verified whether the constructed network can meet the actual needs.

At present, the research on reliability of WSN focuses on the impact of protocols or algorithms on network performance [3-5]. In this paper, we will extract the performance indexes of WSN from the system perspective and establish a wireless sensor network reliability model based on fuzzy neural network to achieve WSN reliability assessment. 


\section{Performance Index of Wireless Sensor Network}

At present, there are no uniform standards for wireless sensor network performance. There are too many wireless sensor network performance indexes; this paper will extract WSN performance indexes from the architecture, topology, and protocol stack structure and reliability mechanism of WSN.

\subsection{WSN architecture}

Wireless sensor network architecture as shown in Figure 1, wireless sensor networks typically include sensor nodes, sink nodes, and management nodes. A large number of sensor nodes are randomly deployed within or near the sensor field and can form networks [6] through self-organizing methods. The monitoring data is collected by the sensor node and transmitted to the sink node, then transmitted to the task management node or database through the network or the base station.

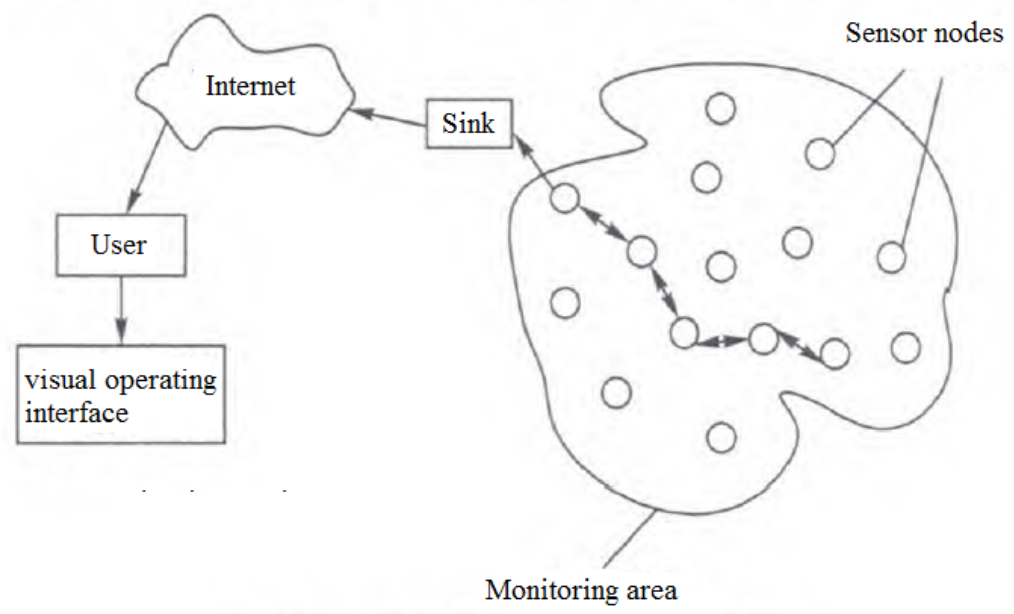

Fig. 1. Wireless sensor network architecture

Each sensor node is equipped with a converter, a microcontroller, a wireless communication module and an energy source. The converter converts the monitored environmental changes into electrical signals. The microcontroller is used to process and manage electrical signals, and the wireless communication module is used to transmit data or communicate with other nodes. Each sensor node doesn't have a fixed position and they are randomly placed in the sensor field.

Wireless sensor networks are mainly used in two topologies: star network topology and point-to-point network topology. In a star network, the formation of the entire network and the transmission of data are controlled by the central network coordinator. Each terminal device directly associates with the network coordinator and transmits 
data. Point-to-point networks are different from star networks. In a point-to-point network, all devices can communicate with all other devices within the range of signal radiation, which makes point-to-point network topologies form more complex forms of networks, such as multi-level cluster tree network, Mesh network, etc.

\subsection{WSN protocol stack structure}

The International Organization for Standardization (ISO) has developed an Open System Interconnection (OSI) seven-layer model to form the basis of the WSN protocol stack design. However, the WSN protocol stack includes the physical layer, data link layer, network layer, transport layer, and application layer. It does not use all layers of the OSI model [7]. In fact, OSI seven-layer model has too many layers to make it complicated and difficult to implement. Therefore, the WSN protocol stack only includes five layers and each layer is assigned a set of tasks independent of other layers of the protocol stack.

The first layer of the protocol stack is the physical layer. It is mainly responsible for defining and managing the connection and communication medium between devices. The physical layer is responsible for frequency selection, carrier frequency generation, signal detection, modulation, and data encryption.

The second layer of the protocol stack is the data link layer and is mainly responsible for providing services for multi-node access and shared communication media. These services include media access control, reliable transmission, error detection, and error correction.

The third layer of the protocol stack is the network layer and is mainly responsible for establishing communication paths between network nodes. Data packets are successfully routed along these paths. The requirements of different routing protocols may vary, and the choice of routing will affect the setting of the communication path. Some routing protocols can establish a favorable communication path to help wireless sensor networks provide the best quality of service.

The fourth layer of the protocol stack is the transport layer and is mainly responsible for providing a higher level protocol stack layer, thereby providing users with transparent and reliable communication. There are different forms of transmission protocols. The two most popular and most comparable transmission protocols are Transmission Control Protocol (TCP) and User Datagram Protocol (UDP). Connection-oriented transport layer protocols (such as TCP) provide reliable communication services with extensive error handling, transmission control, and flow control. However, connectionless transport layer protocols (such as UDP) provide unreliable services with minimal error handling, transmission, and flow control.

The fifth layer of the protocol stack and adopted by a large number of WSN is the application layer. The application layer is close to the user of the system; there are many potential applications at the application layer, including Telnet, Hyper Text Transfer Protocol (HTTP), File Transfer Protocol (FTP), or Simple Mail Transfer Protocol (SMTP). In the field of WSN, application layer programming mainly involves information processing, data encryption, formatting and storage. In addition, the application 
layer scans its underlying layers to detect if there are enough network resources and services to satisfy the user's network requests.

\subsection{WSN reliability mechanism}

The main reliability mechanisms of WSN include coding and modulation, power control, fault tolerance, error retransmission mechanisms, monitoring/sleep mechanisms, channel access mechanisms, routing strategies, and data fusion mechanisms [8]. Fault tolerance, error retransmission mechanisms, monitoring/sleep mechanisms, channel access mechanisms, and routing strategies were focused on in this paper.

Fault Tolerance: Fault tolerance mainly refers to that when the network fails, WSN can still complete the work normally. The principle of fault tolerance is mainly achieved through device redundancy; the essence of fault tolerance is to improve reliability by sacrificing device resources; although the reliability of the equipment has been greatly improved, for some networks that are not allowed to make mistakes, improving reliability through device redundancy is an important approach. The main performance indexes for measuring fault tolerance are equipment redundancy, channel redundancy, Mean Time Between Failures (MTBF), and the Mean Time To Repair (MTTR).

Monitor/Sleep: The mechanism is mainly to reduce the energy consumption of the sensor nodes and improve the life of the sensor nodes. Especially when the message transmission frequency is low, using the monitor/sleep mechanism can effectively improve the network reliability. However, this mechanism will increase the delay of messages and it will not apply to networks with high real-time requirements.

Channel access: The channel access mainly considers the fairness of the channel access, including whether the time when the network node accesses the channel is fair and whether the bandwidth occupied by the network node is fair. The metrics are the maximum time for the nodes in the cluster to occupy the channel, the shortest time for the nodes in the cluster to occupy the channel, the average time for the nodes to occupy the channel in the cluster, and the average bandwidth occupied by the nodes in the cluster.

Routing policy: The routing policy considers the validity of the route. Its metrics include route outage probability, route signaling overhead, average path length, shortest path length, longest path length, route reconstruction delay, and end-to-end data transmission rate.

Error retransmission: It is an important mechanism to guarantee data reliability. After the node sends data, if no response is received within the specified time, the data is resent; metrics include the maximum number of retransmissions.

\subsection{WSN reliability mechanism}

From the physical layer, the focus is on some performance indexes based on bits of physical devices, such as line connectivity, physical bandwidth, transmission delay, and bit throughput of the interface. 
From the data link layer, the focus is on some performance indexes of the data link based on the frame, such as the link layer frame throughput, link propagation delay, frame loss rate.

From the network layer, the emphasis is on point-to-point or end-to-end based performance metrics, such as network packet throughput, point-to-point or end-to-end connectivity, packet transmission delay, delay jitter, packet loss rate, etc.

For the WSN topology, the star, mesh, and cluster tree networks are mainly considered. The star network is suitable for small networks. It is generally used in home automation, personal computer peripherals, toys, and personal health care. Mesh and cluster tree networks are self-organizing and self-healing. They are suitable for large-scale networks and can implement multi-hop routing for data transmission. They are suitable for industrial control and monitoring, wireless sensor networks, cargo storage and tracking, smart agriculture and security.

Through the analysis of the WSN architecture, topology, protocol stack, and major reliability mechanisms, the WSN performance metrics are divided into two categories: network performance and mechanism strategy. The network performance mainly includes the indexes extracted from the topological structure, the architecture and the protocol stack, and the mechanism policies mainly include the indexes extracted by the reliability mechanism.

In this paper we consider WSN reliability as a primary index, network performance and mechanism policy as secondary indexes, and performance indexes as tertiary indexes.

\section{WSN reliability model}

Based on the fuzzy neural network, WSN performance indexes were used to establish a WSN reliability evaluation model at the system level.

\subsection{WSN reliability mechanism}

Fuzzy neural network is a new technology [9] that integrates the structural knowledge expression ability of fuzzy logic inference and the learning ability of neural network. In general, the fuzzy neural network mainly uses the neural network structure to achieve fuzzy logic reasoning, so that the weights that have no explicit meaning in traditional neural networks are given the physical meaning of inference parameters in fuzzy logic.

As shown in Figure 2, the fuzzy neural network model based on Mamdani reasoning consists of five layers. 


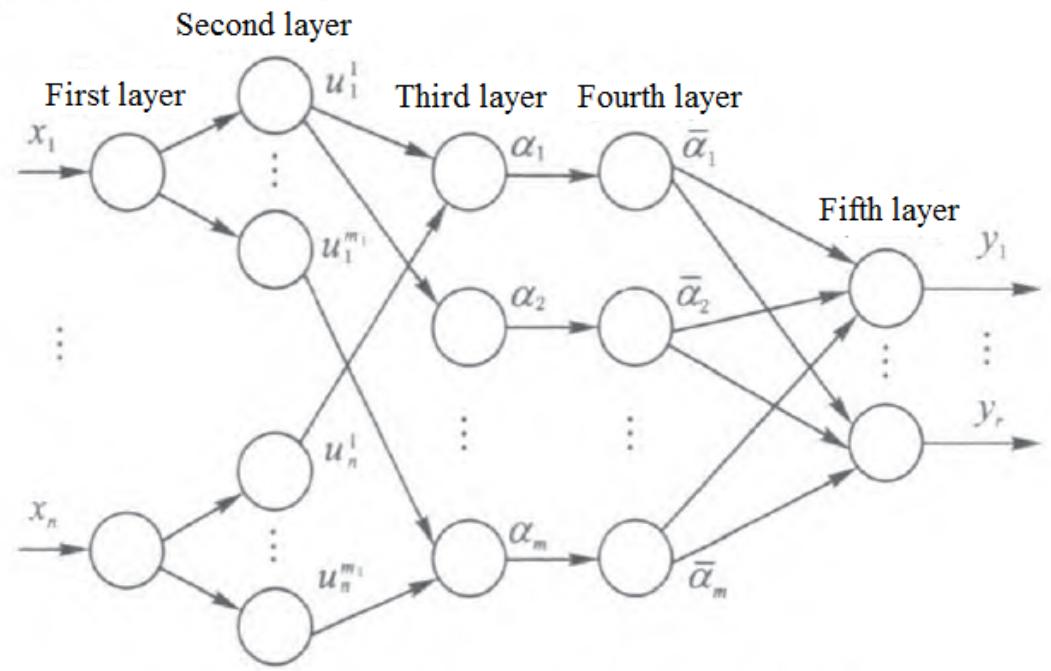

Fig. 2. The structure of fuzzy neural network based on Mamdani reasoning

The first layer is the network input layer, which inputs the evaluation index to the next layer. The number of nodes in this layer is $\mathrm{N}_{-} 1=\mathrm{n}$, where $\mathrm{n}$ is the number of indexes. The second layer is the fuzzy layer of the network. Each node represents a linguistic variable value, such as Negative Big (NB), Positive Big (PB), and so on. In the second layer, the first-input index values are fuzzy quantified using membership functions [10], and the membership functions which each input index value belongs to a fuzzy set of value of each language variable are calculated. The number of nodes in this layer is $N_{2}=\sum_{i=1}^{n} m_{i}$, where $m_{-} i$ denotes the number of fuzzy divisions of the $i-t h$ index. Each node in the third level represents a fuzzy rule that is used to match the front of the fuzzy rule and calculate the degree of applicability of each rule, using the following formulas

$$
\alpha_{\mathrm{j}}=\min \left\{\mathrm{u}_{1}^{\mathrm{i} 1}, \mathrm{u}_{2}^{\mathrm{i} 2}, \ldots, \mathrm{u}_{\mathrm{n}}{ }^{\mathrm{in}}\right\}
$$

or

$$
\alpha_{j}=u_{1}{ }^{i 1}, u_{2}{ }^{i 2}, \ldots, u_{n}{ }^{i n}
$$

where $i_{1}=\left\{1,2, \ldots, m_{1}\right\} \quad, \quad i_{2}=\left\{1,2, \ldots, m_{2}\right\}, \ldots, \quad i_{n}=\left\{1,2, \ldots, m_{n}\right\}, \quad j=$ $\{1,2, \ldots, m\}, m=\prod_{i=1}^{n} m_{i}$. The total number of nodes in this layer $N_{3}=m$. The fourth layer has the same number of nodes as the third layer, $N_{4}=N_{3}=m$, it implements a normalized calculation, which can be defined as follows:

$$
\bar{\alpha}_{\mathrm{j}}=\alpha_{\mathrm{j}} / \sum_{\mathrm{j}=1}^{\mathrm{m}} \alpha_{\mathrm{j}} ; \mathrm{j}=1,2, \ldots, \mathrm{m}
$$

The fifth layer is the output layer, also called anti-fuzzy layer, which achieves clear calculations and the number of nodes is the target value [11-12]. 


\subsection{Fuzzy quantification for evaluation index}

Assuming that WSN reliability evaluation is divided into q levels and there are $\mathrm{m}$ performance indexes, the q-level evaluation corresponding to these $m$ evaluation factors constitutes the reliability evaluation standard value matrix:

$$
C=\left(c_{i j}\right)_{m \times q}
$$

where, $c_{i j}$ indicates that the performance index of item i corresponds to the standard value of level $j$.

In this paper, WSN reliability evaluation is divided into four levels, and 14 performance indexes are selected. These indexes make up the reliability evaluation standard value matrix. The reliability standard value matrix $C$ represents the standard value of each reliability index corresponding to each reliability level, and the setting of the standard value satisfies the positive correlation or negative correlation between the performance index and the reliability; because wireless sensor networks are used in life, industry, and military applications, the performance requirements of wireless sensor networks are different. It is not possible to have a common standard value to establish a reliability standard value matrix. The reliability standard value matrix given in this paper does not strictly give the standard values that can be applied in practice, but instead provides the standard values that satisfy the evaluation of general WSN. The reliability standard value matrix can be used in practical applications according to the needs of specific settings.

The relative membership degree in fuzzy mathematics is used to transform each reliability performance index data into the relative membership degree of the evaluation hierarchy, the relative membership degree matrix $R=\left(\mathrm{r}_{\mathrm{ij}}\right)_{\mathrm{m} \times \mathrm{q}}$ of the detected sample index is obtained. For positive correlation performance indexes, when $x_{i j} \leq c_{i 1}, r_{i j}=$ 0 , when $x_{i j} \geq c_{i q}, r_{i j}=1$. For negative correlation performance indexes, when $x_{i j} \geq$ $\mathrm{c}_{\mathrm{i} 1}, \mathrm{r}_{\mathrm{ij}}=0$, when $\mathrm{x}_{\mathrm{ij}} \leq \mathrm{c}_{\mathrm{iq}}, \mathrm{r}_{\mathrm{ij}}=1$. When between $\mathrm{c}_{\mathrm{i} 1}$ and $\mathrm{c}_{\mathrm{iq}}, \mathrm{r}_{\mathrm{ij}}$ is:

$$
r_{i j}=\left(x_{i j}-c_{i 1}\right) /\left(c_{i q}-c_{i 1}\right)
$$

\subsection{Sample data selection}

In order to improve the training accuracy, a limited number of interpolations are performed on the reliability evaluation standard value matrix $C$, and the index i defining the generated sample $\mathrm{k}$ has the value $\mathrm{c}_{\mathrm{kij}}$ for the evaluation level $\mathrm{j}$, and the standard level value corresponding to the interpolation sample $\mathrm{k}$ is $\mathrm{T}_{\mathrm{k}}$, then:

$$
\begin{gathered}
c_{k i j}=c_{i j}+\left(c_{i(j+1)}-c_{i j}\right) t /(n+1) \\
T_{k}=|j+t /(n+1)|
\end{gathered}
$$

where $=1,2, \ldots, \mathrm{m} ; \mathrm{j}=1,2, \ldots, \mathrm{q} ; \mathrm{t}=1,2, \ldots, \mathrm{n}-1 ; \mathrm{n}$ represents the number of samples inserted between two reliability levels. In this paper, $n=5$. By using the above formula, a total of 15 sets of sample data was generated. 


\subsection{Sample data selection}

The reliability evaluation model of WSN established in this paper is a 5-layer fuzzy neural network model. The network structure of the model is shown in Figure 2. It consists of one input layer, three middle layers and one output layer. The input layer has 14 nodes, each node corresponds to a performance index, the output layer has three nodes, which represent the reliability level of the evaluated wireless sensor network, and the target value of the output result is:

$$
\left[\begin{array}{l}
0 \\
0 \\
1
\end{array}\right],\left[\begin{array}{l}
0 \\
1 \\
0
\end{array}\right],\left[\begin{array}{l}
0 \\
1 \\
1
\end{array}\right],\left[\begin{array}{l}
1 \\
1 \\
0
\end{array}\right]
$$

The four output matrices represent the four levels of reliability assessment (excellent, good, medium, and poor).

The trained matrix model and the target matrix training model are used to store the trained network model. The established model program flow is shown in Figure 3.

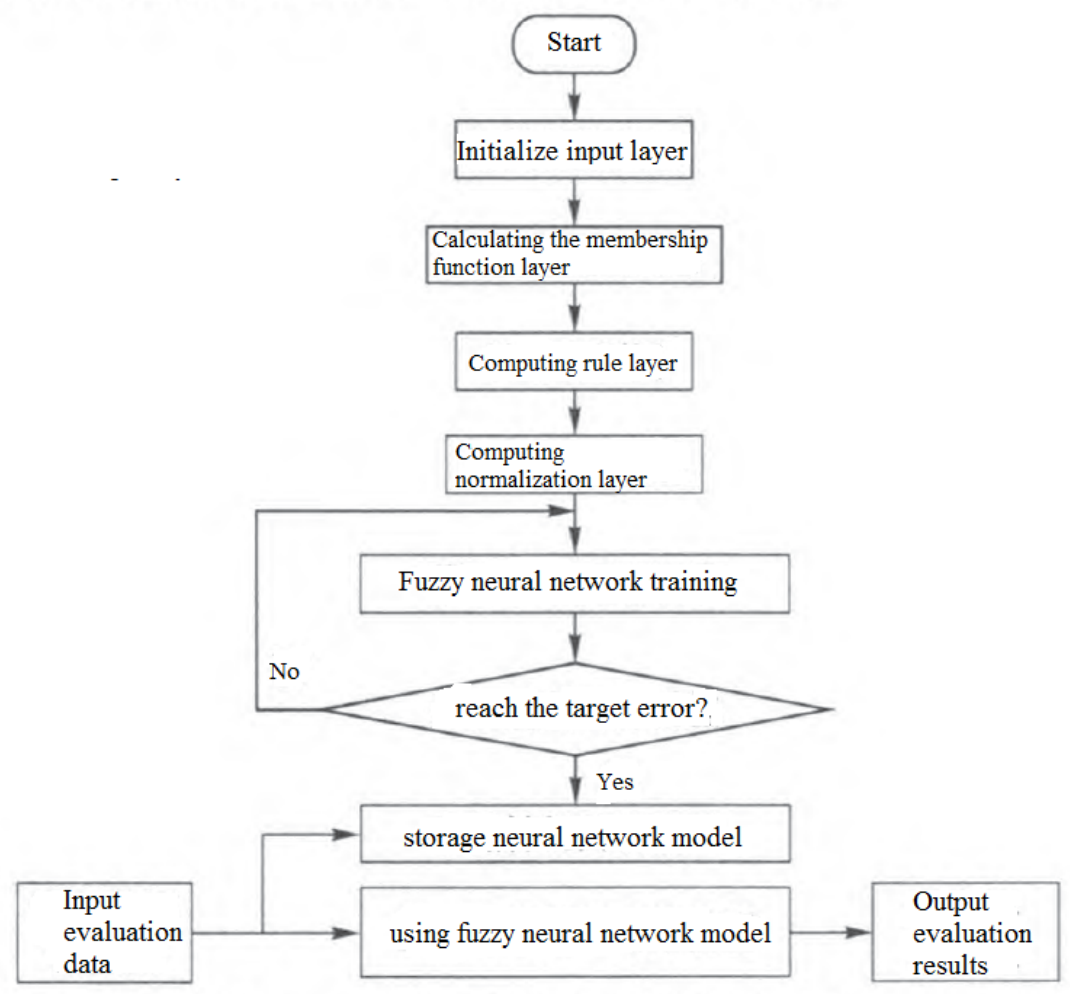

Fig. 3. Flow chart of reliability model for Wireless Sensor Networks 


\section{$4 \quad$ Experimental Results and Analysis}

A simulation program was written according to the model program flow. The model was trained using 15 sample data generated by interpolation. When the network error was close to 0 , the training was stopped. The network's expected error value is 0.001 . After 715 trainings, the expected error value is reached, the training stops and a suitable network model is obtained through training. The training process is shown in Figure 4.

The sample corresponding to the reliability evaluation standard value matrix is input as a test sample into the trained network model for testing. The output result is the reliability of the WSN corresponding to the index. The test results are shown in Table 1.

Table 1. Table 1. Test result

\begin{tabular}{|c|c|c|c|c|}
\hline Test sample & Reliability model output & Test result & Expected output & Evaluation matrix \\
\hline 1 & {$\left[\begin{array}{llll}0.005 & 0.098 & 0.9821\end{array}\right]^{T}$} & excellent & excellent & {$\left[\begin{array}{lll}0 & 0 & 1\end{array}\right]^{T}$} \\
\hline 2 & {$\left[\begin{array}{llll}0.096 & 0.218 & 0.9651\end{array}\right]^{T}$} & excellent & good & {$\left[\begin{array}{lll}0 & 1 & 0\end{array}\right]^{T}$} \\
\hline 3 & {$\left[\begin{array}{lllll}0.033 & 0.999 & 0.9988\end{array}\right]^{T}$} & medium & medium & {$\left[\begin{array}{llll}0 & 1 & 1\end{array}\right]^{T}$} \\
\hline 4 & {$\left[\begin{array}{llllll}0.998 & 0.008 & 0.0109\end{array}\right]^{T}$} & poor & poor & {$\left[\begin{array}{lll}1 & 0 & 0\end{array}\right]^{T}$} \\
\hline
\end{tabular}

According to the test results, the evaluation results of the established WSN reliability evaluation model are basically consistent with the actual situation, which prove that this model is more reliable. From Table 1, we can find that the test result of sample 2 is inconsistent with the expected output result. It is due to the fact that the training sample data used in this paper is less, and the training samples used are mostly obtained through interpolation. However, it doesn't affect the reliability of the model. In practical applications, a large number of WSN index data can be collected to train the reliability model, which can greatly improve the accuracy of the assessment of the network.

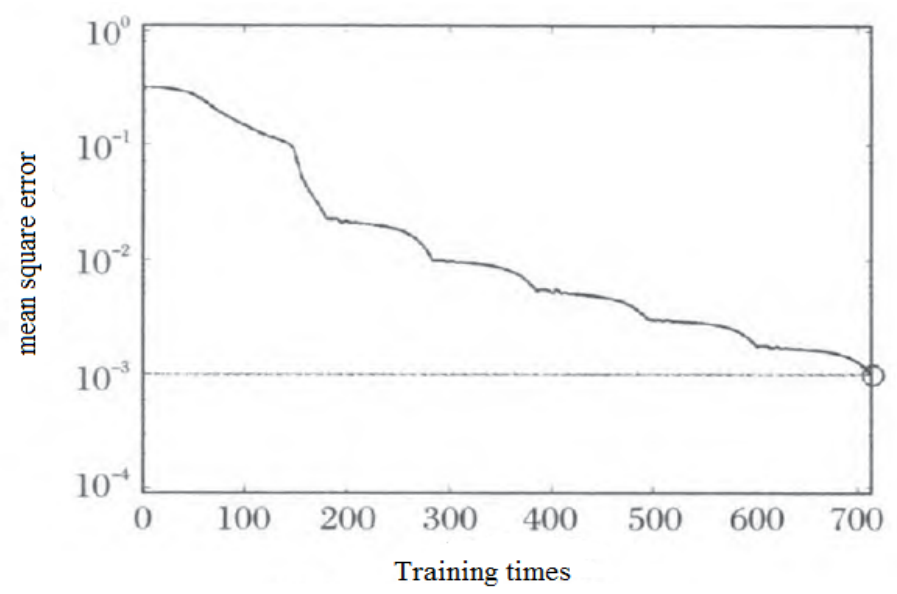

Fig. 4. Network training process 
The node reliability function $\mathrm{R}(\mathrm{t})$ indicates the probability of the sensor node being in working state at the time $t$. No matter what function distribution $R(t)$ obeys, WSN reliability function should have a critical threshold. The reliability functions distribution diagram of nodes that obey exponential distribution and even distribution are shown in Figures 5 and Figures 6 respectively.

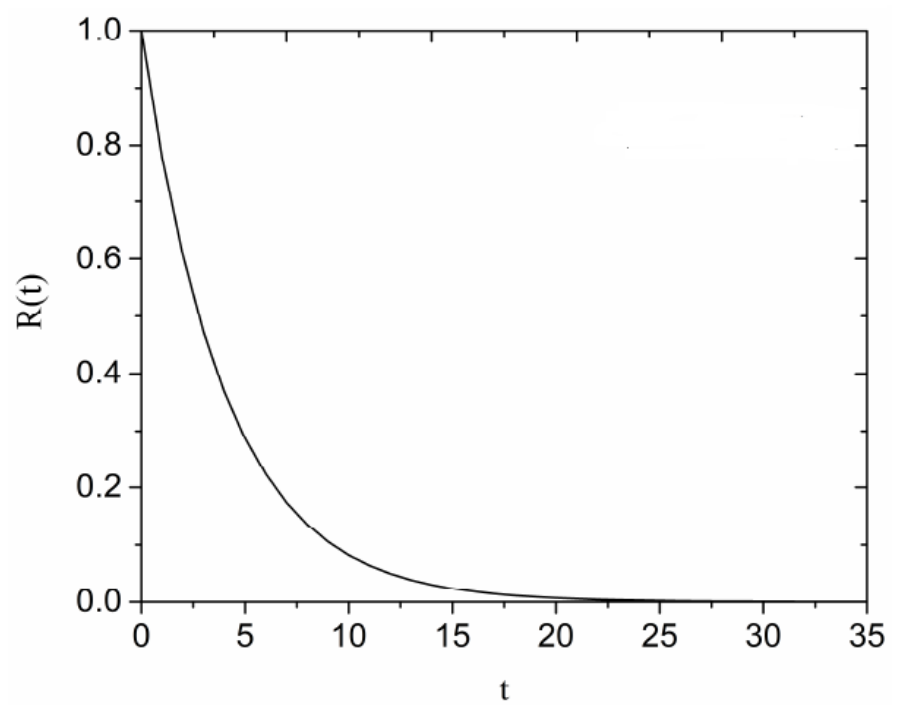

Fig. 5. Reliability functions distribution diagram of nodes that obey exponential distribution

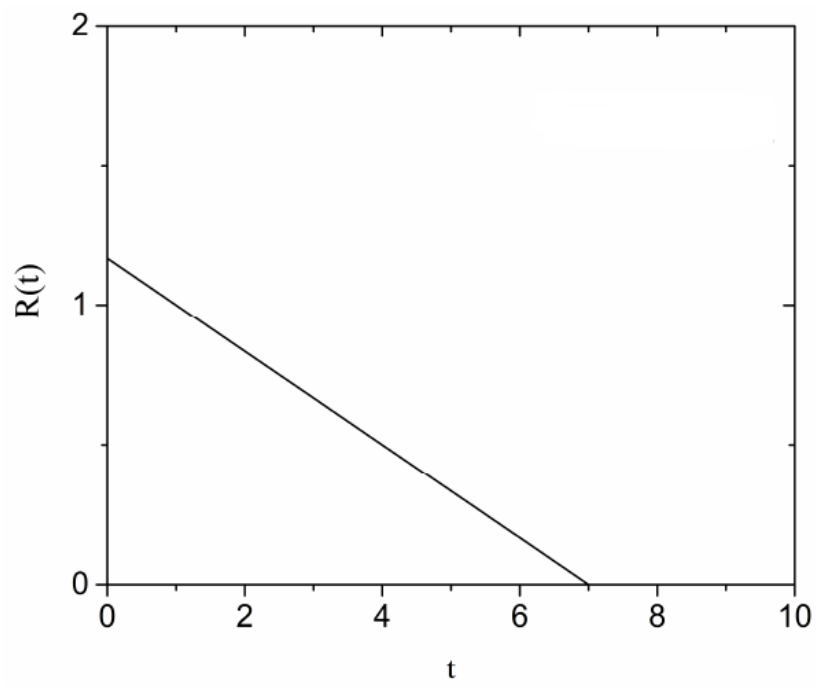

Fig. 6. Reliability functions distribution diagram of nodes that obey even distribution 
Suppose a WSN consisting of 100 identical sensor nodes. The average degree is $\langle\mathrm{k}\rangle=4$. According to node reliability distribution $\mathrm{R}(\mathrm{t})$, which conforms to exponential distribution and uniform distribution, WSN reliability $R_{s}(t)$ can be obtained when two different distributions are used, as shown in Figures 7 and 8 respectively.

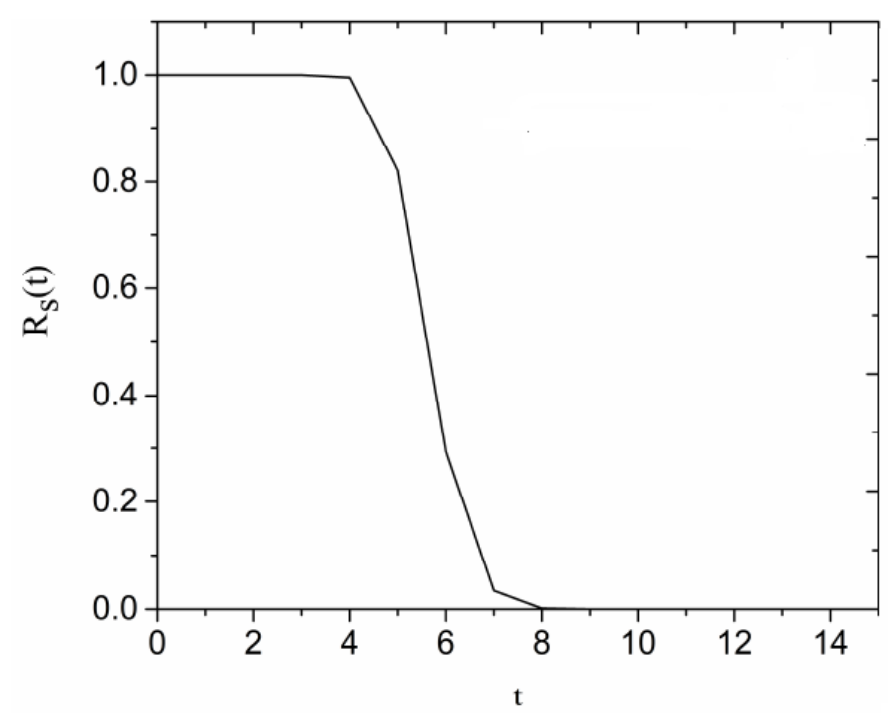

Fig. 7. Reliability of Wireless Sensor Networks when nodes obey exponential distribution

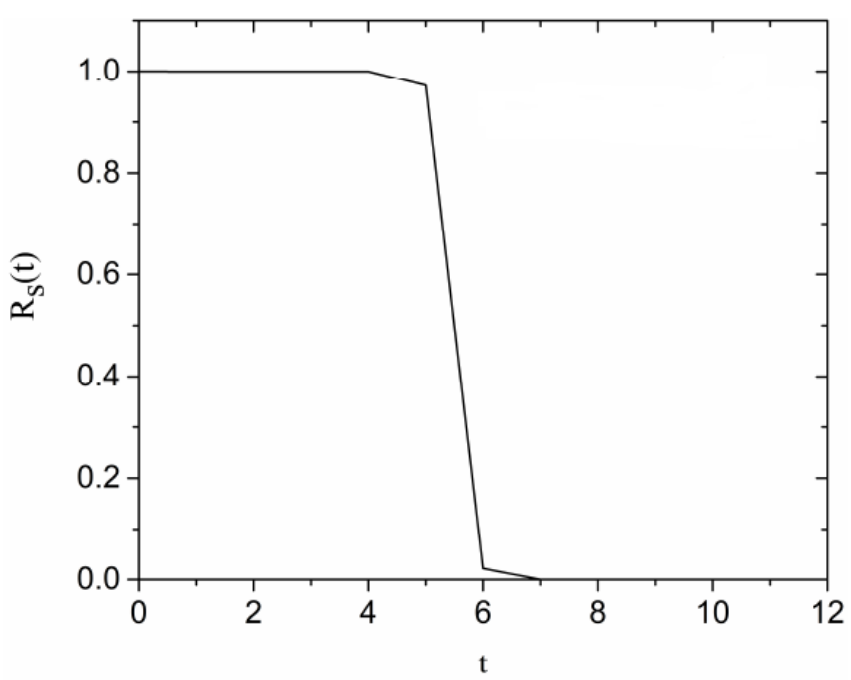

Fig. 8. Reliability of Wireless Sensor Networks when nodes obey even distribution

From Figure 7 and Figure 8 we can see that there exists the critical threshold of WSN reliability, when WSN work time reaches a certain time, the reliability of WSN drops 
sharply. Comparison between Figure 7 and Figure 8, we found that no matter what the reliability of sensor nodes fits, there is a critical value for WSN reliability. The simulation results show that the WSN reliability function $R_{S}(t)$ is general, and is not directly related to the node reliability $R(t)$. The characteristic can be used to evaluate the reliability of WSN.

The reliability indexes used to evaluate the wireless sensor network is extracted from the system level and not only considers the network lifetime, network connectivity, protocols and algorithms. This paper introduces the concept of positive correlation and negative correlation indicators, taking into account the mutual constraints between various performance indicators, making the assessment results more accurate and comprehensive. Existing assessment techniques consider only a single factor or several factors and are targeted in reliability assessment. For a WSN that only pays attention to the network survival time or network connectivity, the evaluation technology using a single factor is more effective; for considering the overall reliability of the wireless sensor network, the evaluation technology proposed in this paper is more effective.

\section{Conclusions}

This paper extracts the performance indexes of WSN from the perspective of topology, protocol stack structure and reliability mechanism, and filters 14 performance indexes from them, and establishes a WSN reliability evaluation model based on fuzzy neural network. After testing, the evaluation results of the model are basically consistent with the actual situation. It makes the WSN reliability evaluation effectively achieved. Further research work is to increase the consideration of the field environment of WSN, further explore the mutual constraints between performance indicators, refine the reliability assessment level, and establish a wireless sensor network reliability assessment tool based on the final model.

\section{References}

[1] Brak M E, Brak S E, Essaaidi M. (2015). Wireless Sensor Network applications in smart grid. Renewable and Sustainable Energy Conference. IEEE, pp 587-592.

[2] Basu D, Moretti G, Gupta G S. (2013). Wireless sensor network based smart home: Sensor selection, deployment and monitoring. Sensors Applications Symposium. IEEE, pp 49-54. https://doi.org/10.1109/SAS.2013.6493555

[3] Jose D V, Sadashivappa G. (2015). Mobile Sink Assisted Energy Efficient Routing Algorithm for Wireless Sensor Networks. World of Computer Science \& Information Technology Journal, 5(2).

[4] Chan H F, Rudolph H. (2016). New energy efficient routing algorithm for Wireless Sensor Network. TENCON 2015 - 2015 IEEE Region 10 Conference. IEEE, pp 1-5.

[5] Shang J, Liu C, Yue K. (2015). Node Location of Wireless Sensor Networks Based on Improved Two-system Cooperative Evolutionary Algorithm. Computer Application, 35(6): 1514-1518.

[6] Sun L. (2005). Wireless sensor network. Tsinghua University Press, pp 3-10. 
[7] Yang S H. (2014). Wireless Sensor Networks: Principles, Design and Applications. Springer Publishing Company, Incorporated. https://doi.org/10.1007/978-1-4471-5505-8

[8] Yick J,Mukheiuee B,Ghosal D. (2008). Wireless sensor network survey. Computer Networks, 52(12): 2292-2330. https://doi.org/10.1016/j.comnet.2008.04.002

[9] Fan H. (2006). Application of MATLAB Neural Network and Optimization Toolbox in Design Optimization. Computer Engineering \& Applications, 42(16): 187-189.

[10] Zadeh L A. (1978). Fuzzy sets as a basis for a theory of possibility. Fuzzy Sets and Systems, 1(1): 3-28. https://doi.org/10.1016/0165-0114(78)90029-5

[11] Kuo R J. (2007). A sales forecasting system based on fuzzy neural network with initial weights generated by genetic algorithm. European Journal of Operational Re-search, 129(3): 496-517. https://doi.org/10.1016/S0377-2217(99)00463-4

[12] Long X, Wang J, Chen Q. (2012). Kalman filtering state of charge estimation for battery management system based on a stochastic fuzzy neural network battery model. Energy Conversion \& Management, 53(1): 33-39. https://doi.org/10.1016/j.enconman.2011.06.003

\section{$7 \quad$ Author}

Jianjun Xu is a member of the Taizhou College of Nanjing Normal University, 518 Dongfeng Road, Taizhou, Jiangsu. His research fields are mainly about remote sensing spectrum, information technology, geographic information system and so on.

Article submitted 15 July 2018. Resubmitted 28 July 2018. Final acceptance 13 September 2018. Final version published as submitted by the author. 